\title{
Targeted removal of copper foil surface impurities for improved
}

\section{synthesis of CVD graphene}

Adrian T. Murdock ${ }^{1,2}$, Christian D. van Engers ${ }^{1}$, Jude Britton ${ }^{1,3}$, Vitaliy

Babenko $^{1,4}$, Seyyed Shayan Meysami ${ }^{1}$, Hugh Bishop ${ }^{1}$, Alison Crossley ${ }^{1}$, Antal

A. $\operatorname{Koos}^{1,5}$, and Nicole Grobert*1

1. Department of Materials, University of Oxford, Parks Road, Oxford, OX1 3PH, U.K.

2. Present address: CSIRO Manufacturing, Lindfield, NSW, 2070, Australia

3. Present address: Renishaw New Mills, Wotton-under-Edge, Gloucestershire, GL12 8JR, UK

4. Present address: Centre for Advanced Photonics and Electronics, University of Cambridge, 9 JJ Thomson Ave, Cambridge, CB30FA, UK

5. Present address: Institute of Technical Physics and Materials Science, Centre for Energy Research 1525 Budapest, P.O. Box 49, Hungary

* Corresponding author. Tel: +44 18652 83720. E-mail: nicole.grobert@materials.ox.ac.uk (Professor Nicole Grobert) 


\begin{abstract}
Commercially available $\mathrm{Cu}$ foils are leading candidates as substrates employed for the generation of large-area graphene using chemical vapour deposition (CVD) techniques. However, the growth of high-quality graphene on $\mathrm{Cu}$ foils is often hindered by contamination particles, which will also be detrimental for many potential applications of graphene. Here we investigate the influence of typical substrate impurities on the formation of CVD graphene using as-received $\mathrm{Cu}$ foils of various purities from different suppliers and the same cleaned by popular methods. Analytical characterisation of the $\mathrm{Cu}$ foils revealed that contamination particles consist of calcium, aluminium, and silicon oxides. We show that contamination particles are present on foils with purities ranging between $99.8 \%$ and $99.9999 \%$ and that these particles influence the nucleation density, growth rate, and growth features of graphene domains. Based on our findings we propose new industrially applicable targeted cleaning procedures of immersion in purposely-selected $\mathrm{HCl}$ and $\mathrm{KOH}$ solutions to chemically dissolve the aforementioned impurities, bringing about improved growth of graphene.
\end{abstract}




\section{Introduction}

Chemical vapour deposition (CVD) of carbon-containing precursors (commonly $\mathrm{CH}_{4}$ ) is widely recognised as the likely manufacturing process for the production of large-area graphene [1,2]. While many substrates have been demonstrated to be suitable for the generation of CVD graphene, metals substrates [3] - in particular, commercially available $\mathrm{Cu}$ foils [4]- have received significant interest as they are lowcost, readily obtainable, scalable, and compatible with roll-to-roll processing [5-7]. $\mathrm{Cu}$ substrates catalytically assist precursor decomposition and simultaneously provide the support for surface-mediated graphene growth. Consequently, the properties and quality of the $\mathrm{Cu}$ foil significantly influence the growth of CVD graphene. Differences will exist between different $\mathrm{Cu}$ foils due to natural variations within the raw material and the influence of processing by suppliers. Key properties that may vary include:

i. Surface topography and roughness,

ii. Surface composition, contamination particles and coatings,

iii. Bulk composition and dissolved impurities,

iv. Texture (crystallographic orientation distribution),

v. Grain sizes.

These properties may have wide ranging influences. For instance, surface roughness can influence the nucleation density of graphene domains [8-10]; surface composition, particularly oxygen and carbon species $[11,12]$, influence nucleation density, while surface contamination particles can also act as a catalyst [13] or inhibitor/etchant [1416] to influence growth rates, or promote multilayer growth [17], or increase the nucleation density often with inhomogeneity [18]; dissolved impurities may migrate to the surface during annealing and so also have capacity to influence growth; crystallographic orientations can influence growth rates [19] or control the alignment 
of graphene domains [20]; while grain boundaries can increase the nucleation density [9].

The ground-breaking work by Li et al. [4] used 99.8 \% purity Alfa Aesar Cu foil (item number 13382) as-received from the supplier. As a consequence, and most likely for consistency alone, many investigations used this particular substrate [16, 21-31]. More expensive high-purity $\mathrm{Cu}$ foils have also been used [29, 32-35], but the benefits of these over the relatively inexpensive, low purity counterpart remains unclear, especially because contamination particles have been frequently observed on the surface of both low and high purity $\mathrm{Cu}$ foils. Zhang et al. [36] first stated that the 99.8 $\%$ Alfa Aesar $\mathrm{Cu}$ foils were coated by the supplier with a layer of chromium oxide, but supporting analytical studies were not provided. In contrast, Kim et al. [37] reported that particles present on the same foil were mostly metals, e.g. $\mathrm{Si}, \mathrm{Ca}, \mathrm{Pt}, \mathrm{Ru}$, and $\mathrm{Ce}$, however, the exact origin, precise composition, and variability of the particles were not investigated. While Gan and Luo [31] again used the same foil, they claimed the surface nanoparticles may in fact be $\mathrm{Cu}$-oxide or $\mathrm{Cu}$, depending on whether the foil was annealed in $\mathrm{Ar}$ or $\mathrm{Ar} / \mathrm{H}_{2}$. Fan et al. [14] reported that similar nanoparticles on $\mathrm{Cu}$ foils are composed of $\mathrm{Si}$, or composites of $\mathrm{Fe}, \mathrm{Al}, \mathrm{Si}, \mathrm{Ca}, \mathrm{O}$ and $\mathrm{C}$, but purity, thickness, and supplier details of the $\mathrm{Cu}$ foil were not provided. Recently, Wang et al. [38] showed the nucleation of $\mathrm{SiO}_{2}$ nanoparticles by heating $\mathrm{Cu}$ foils in situ using scanning electron microscopy (SEM). Whilst these findings are interesting, the analyses cannot be generalised for all $\mathrm{Cu}$ foils, especially those with different purities, and the range of opposing and conflicting studies necessitates more in-depth investigations. An accurate understanding of the nature of the particles, and development of an efficient procedure for their removal is imperative for reproducible graphene growth, and production of particle-free graphene for future applications. A 
variety of procedures have been proposed to clean $\mathrm{Cu}$ foils including treatment with $\mathrm{CH}_{3} \mathrm{COOH}[39,40], \mathrm{FeCl}_{3}[31], \mathrm{HNO}_{3}$ [37], and electropolishing [8, 18, 36]. While cleaning with $\mathrm{CH}_{3} \mathrm{COOH}$ has been replicated in a number of publications $[8,34,37$, $41,42]$ a thorough analysis of the influence of the treatment on the concentration of contaminations on $\mathrm{Cu}$ foils, and the associated growth of graphene on $\mathrm{CH}_{3} \mathrm{COOH}$ cleaned foils, has not been reported. None of these cleaning methods are ideal, with the most effective only etching the $\mathrm{Cu}$ surface rather than addressing the contamination particles. To date, no treatment has been proposed with the aim of chemically dissolving the contamination nanoparticles.

Therefore, despite significant development in recent years, the growth of highquality CVD graphene and its applications are still hindered by contamination particles. For the specific aim of developing targeted chemical cleaning procedures, systematic analysis of different $\mathrm{Cu}$ foils and detailed chemical analysis of surface contamination particles is essential.

In this article we investigate this problem by analytically characterising $\mathrm{Cu}$ foils sourced from different suppliers and with varying purity, and examining the atmospheric pressure CVD (APCVD) growth of graphene on these substrates. The effectiveness of common cleaning treatments that rely on etching of the $\mathrm{Cu}$ foil are evaluated as a benchmark and compared with new cleaning treatments we propose which chemically dissolve the surface contamination particles. We demonstrate that a combination $\mathrm{HCl} / \mathrm{KOH}$ treatment achieves comparable cleaning to electropolishing and provides improved growth of graphene with reduced nanoparticle contamination. Our new, effortless cleaning process is scalable and compatible with roll-to-roll processing and therefore attractive for significantly improving current syntheses of large-area CVD graphene. 


\section{Experimental Methods}

\subsection{Details of $\mathrm{Cu}$ foils}

Six commercially-available $\mathrm{Cu}$ foils were obtained with a range of purities $(99.8 \%$ 99.9999 \%) and from different suppliers (Alfa Aesar and Goodfellow), summarised in Table 1. The labels listed are used herein to distinguish between the foils. AA-99.8C was first used by Li et al. [4] and others. Since then it has been annotated as "coated" by the supplier, Alfa Aesar. To our knowledge the exact details on the nature of this coating are not published.

Table 1: Details of $\mathrm{Cu}$ foils

\begin{tabular}{|c|c|c|c|c|c|}
\hline Label & Purity (\%) & Brand & Description & Thickness $(\boldsymbol{\mu m})$ & Item number \\
\hline AA-99.8C & 99.8 & Alfa Aesar & Coated & 25 & 13382 \\
\hline AA-99.8U & 99.8 & Alfa Aesar & Uncoated; annealed; for graphene growth & 25 & 46986 \\
\hline AA-99.99 & $99.99+$ & Alfa Aesar & - & 127 & 00095 \\
\hline AA-99.999 & 99.999 & Alfa Aesar & Puratronic $^{\oplus}$ & 25 & 10950 \\
\hline AA-99.9999 & 99.9999 & Alfa Aesar & Puratronic $^{\oplus}$ & 50 & 42972 \\
\hline GF-99.9 & 99.9 & Goodfellow & Annealed & 25 & $375-907-90$ \\
\hline
\end{tabular}

\subsection{Chemical treatments of $\mathrm{Cu}$ foils}

The six $\mathrm{Cu}$ foils were annealed for 30 minutes at $1035^{\circ} \mathrm{C}$ under $500 \mathrm{sccm} \mathrm{H}_{2}$ prior to characterisation so that the surfaces were as comparable as possible to foils used for the growth of graphene.

The as-received $\mathrm{Cu}$ foils were also cleaned using a range of chemical treatments:

a) $\mathrm{CH}_{3} \mathrm{COOH}$ treatment- ultrasonicated in concentrated $\mathrm{CH}_{3} \mathrm{COOH}$ (Sigma Aldrich, ReagentPlus ${ }^{\circledR}>99 \%$ ) for 10 minutes

b) $\mathrm{HNO}_{3}$ treatment- ultrasonicated in isopropyl alcohol (Sigma Aldrich, ACS reagent, $\geq 99.8 \%$ ) for 5 minutes, then immersed in 1:5 $\mathrm{HNO}_{3}: \mathrm{H}_{2} \mathrm{O}$ (Sigma Aldrich, ACS Reagent) for 5 minutes.

c) Electropolishing- ultrasonicated in isopropanol (Sigma Aldrich, ACS reagent $\geq$ $99.8 \%$ ) for 5 minutes, followed by electropolishing in a degassed solution of 
$100 \mathrm{~mL}$ deionized water, $50 \mathrm{~mL}$ orthophosphoric acid (Sigma Aldrich, ACS reagent, $\geq 85$ wt. $\%$ in $\mathrm{H}_{2} \mathrm{O}$ ), $50 \mathrm{~mL}$ ethanol (Fischer Scientific UK, analytical reagent grade), $10 \mathrm{~mL}$ IPA, $1 \mathrm{~g}$ urea (Sigma Aldrich, ACS reagent, 99.0 - 100.5 $\%)$ at $4.5 \mathrm{~V}$ for 1 minute [18].

d) $\mathrm{HCl}$ treatment- immersed in $2 \mathrm{M} \mathrm{HCl}$ (Sigma Aldrich, ACS Reagent) for 10 minutes, followed by a rinse in deionised water, and dried under flowing $\mathrm{N}_{2}$.

e) $\mathrm{KOH}$ treatment- immersed in $2 \mathrm{M} \mathrm{KOH}$ (Sigma Aldrich, reagent grade $90 \%$ flakes) for 10 minutes.

f) $\mathrm{HF}$ treatment- immersed in 1:10 HF: $\mathrm{H}_{2} \mathrm{O}$ (Microchem Systems) for 15 minutes. Following these treatments applied separately to the $\mathrm{Cu}$ foils, the $\mathrm{Cu}$ foils were rinsed or ultrasonicated in deionised water and dried under flowing $\mathrm{N}_{2}$.

\subsection{Synthesis of APCVD graphene}

Graphene was synthesised by APCVD of $\mathrm{CH}_{4}$ on the $\mathrm{Cu}$ foils listed in Table 1, asreceived and following treatments (a) to (f). The synthesis was conducted both simultaneously (one experiment with all $\mathrm{Cu}$ foils, placed side-by-side) and separately (multiple experiments using one $\mathrm{Cu}$ foil at a time). Results were consistent between these two approaches. The $\mathrm{Cu}$ foils $(c a .15 \mathrm{~mm} \times 25 \mathrm{~mm}$ ) were inserted into a cylindrical quartz tube (inner diameter $20 \mathrm{~mm}$ ) within a hot-walled tube furnace. The samples were kept under a $100 \mathrm{sccm}$ Ar gas flow away from the hot zone of the furnace. The system was then purged for 10 min with $500 \mathrm{sccm} \mathrm{H}_{2}$. The $\mathrm{Cu}$ foils were rapidly heated by shifting to the centre position of the hot zone of the furnace and then annealed at $1035^{\circ} \mathrm{C}$ for 30 minutes under $500 \mathrm{sccm} \mathrm{H}_{2}$. The $\mathrm{H}_{2}$ flow rate was then

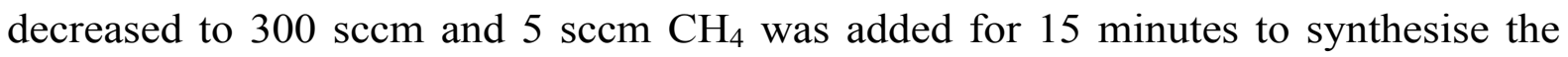
graphene. The $\mathrm{Cu}$ foils were cooled by shifting out of the hot zone and leaving them under a $\mathrm{H}_{2} / \mathrm{CH}_{4}$ flow for 5 minutes, followed by $500 \mathrm{sccm}$ Ar for another 5 minutes. 


\subsection{Characterisation of $\mathrm{Cu}$ foils and APCVD graphene}

SEM was conducted using a JEOL JSM-6500F field emission SEM at $5 \mathrm{kV}$ operating voltage to study the contamination particles, the graphene nucleation sites, the shapes of the graphene domains, and overall coverage of the $\mathrm{Cu}$ foils.

A JY Horiba Labram confocal Raman microscope equipped with a $532 \mathrm{~nm}$ laser was used to determine the quality of the graphene grown on the $\mathrm{Cu}$-foils.

The texture (distribution of crystallographic orientations) of the $\mathrm{Cu}$ foils was studied by electron backscatter diffraction (EBSD) using the SEM operating at $15 \mathrm{kV}$ and an EDAX Digiview II CCD detector. 900 x $900 \mu \mathrm{m}$ areas were mapped and Hough-based analysis was conducted using EDAX OIM ${ }^{\mathrm{TM}}$ Data Analysis software.

Energy dispersive x-ray (EDX) spectroscopy mapping was employed to reveal the chemical composition of contamination particles on the as-received and chemically treated $\mathrm{Cu}$ foils using an Oxford Instruments SSD EDX detector in a JEOL JSM-5510 SEM operating at $15 \mathrm{kV}$ and AZtec EDX analysis software. Both point analysis of $\sim 100 \mathrm{~nm}$ nanoparticles and two-dimensional mapping of micron-sized nanoparticles was performed, with consistent results.

Optical profilometry (OP) of the $\mathrm{Cu}$ foils was conducted using a Nanofocus $\mu$ Surf explorer confocal optical profiler. Average surface area roughness $\left(\mathrm{S}_{\mathrm{A}}\right)$ was calculated from $c a .320 \times 300 \mu \mathrm{m}$ images.

The elemental composition of the surfaces of the as-received and chemically treated $\mathrm{Cu}$ foils was studied using a Thermo Scientific K-Alpha Xray Photoelectron Spectrometer System, and an ion pumped VG Microtech CLAM 4 MCD analyser system equipped with a $200 \mathrm{~W}$ unmonochromated $\mathrm{Mg} \mathrm{K} \alpha$ X-ray radiation of 1253.6 $\mathrm{eV}$, with detection limits of $\sim 0.2$ atomic $\%$. The effectiveness of chemical treatments 
was investigated by conducting detailed XPS line scans over treatment boundaries that were formed by partially submerging the $\mathrm{Cu}$ foils in the cleaning solution.

Secondary ion mass spectrometry (SIMS) was conducted using a MIG300 gallium microprobe and an MM1212 quadrupole mass spectrometer (VG Scientific Ltd.) to investigate the spatial distribution and elemental composition of contamination particles on the $\mathrm{Cu}$ foils. Although SIMS sensitivity varies between different elements, it is very sensitive to many metals in their oxidised state in the positive spectrum, and to oxygen and the halogens in the negative spectrum. Relative concentrations of a particular element between samples was therefore estimated from the ratio of the elements signal intensity to that from the implanted Ga. Elemental depth profiling was also conducted through Ga ion bombardment and simultaneous SIMS.

\section{Results and Discussion}

\subsection{Properties of $\mathrm{Cu}$ foils}

The surface topography, surface impurities, and texture of the six $\mathrm{Cu}$ foils listed in Table 1 after annealing are compared in Figure 1. The influence of these properties on graphene growth is evaluated in later sections. Grain boundaries, surface contamination particles, and residual striations from cold rolling are revealed by SEM and OP. Parallel striations are visible on low purity foils (Figure 1a, 1b, 1c, 1f) but generally absent on the higher purity foils (Figure 1d and 1e) and significantly influence the calculated surface roughness, whereas grain boundaries have less influence. The texture, including grain size and the crystallographic orientations, differs significantly between the six foils, with higher purity foils having fewer grain boundaries as the grain mobility is strongly correlated with the impurity concentration. 
This correlation suggests that the bulk purity of the $\mathrm{Cu}$ foils varies as specified by the suppliers.
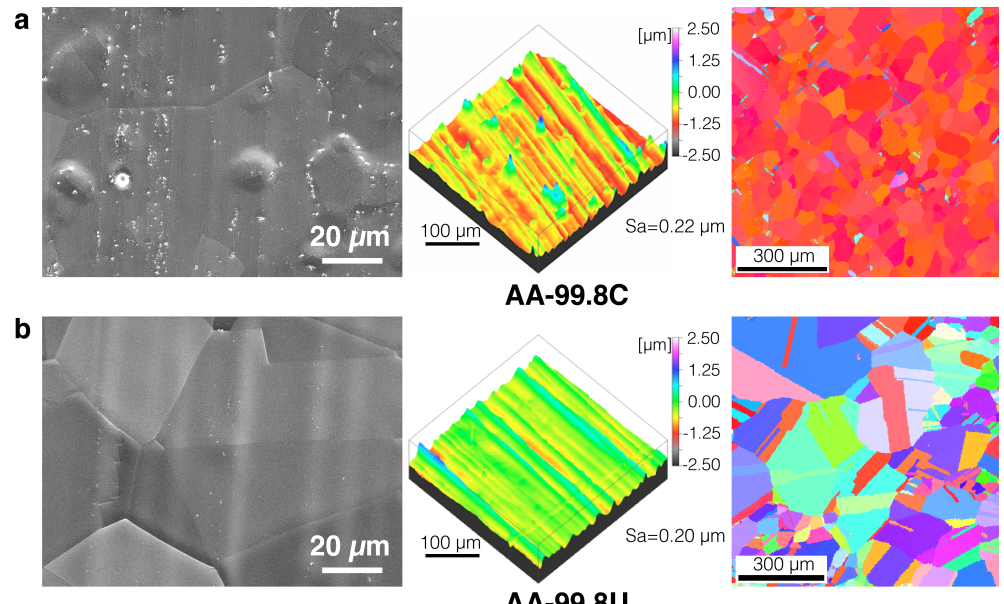

c
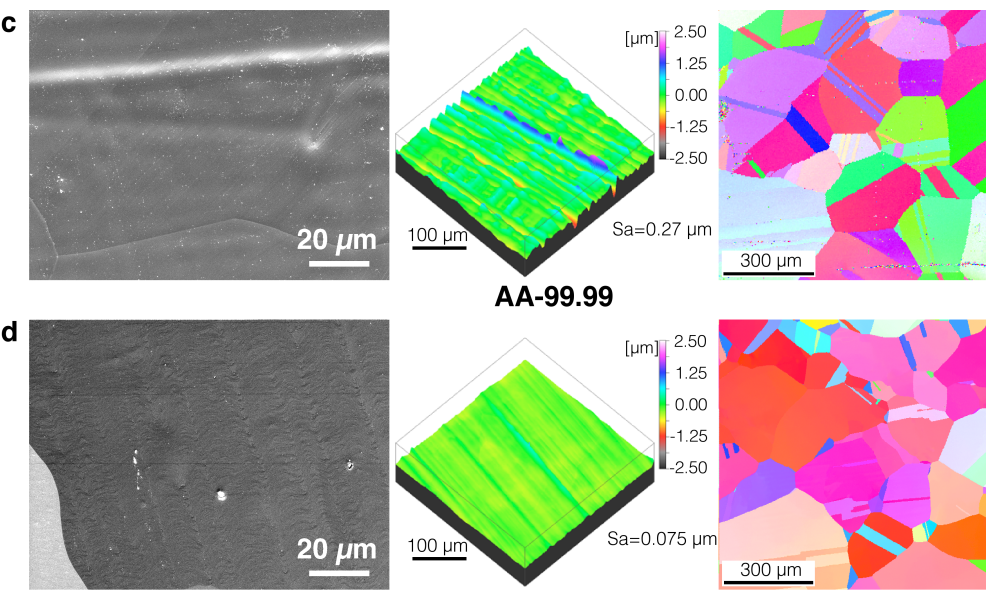

AA-99.99
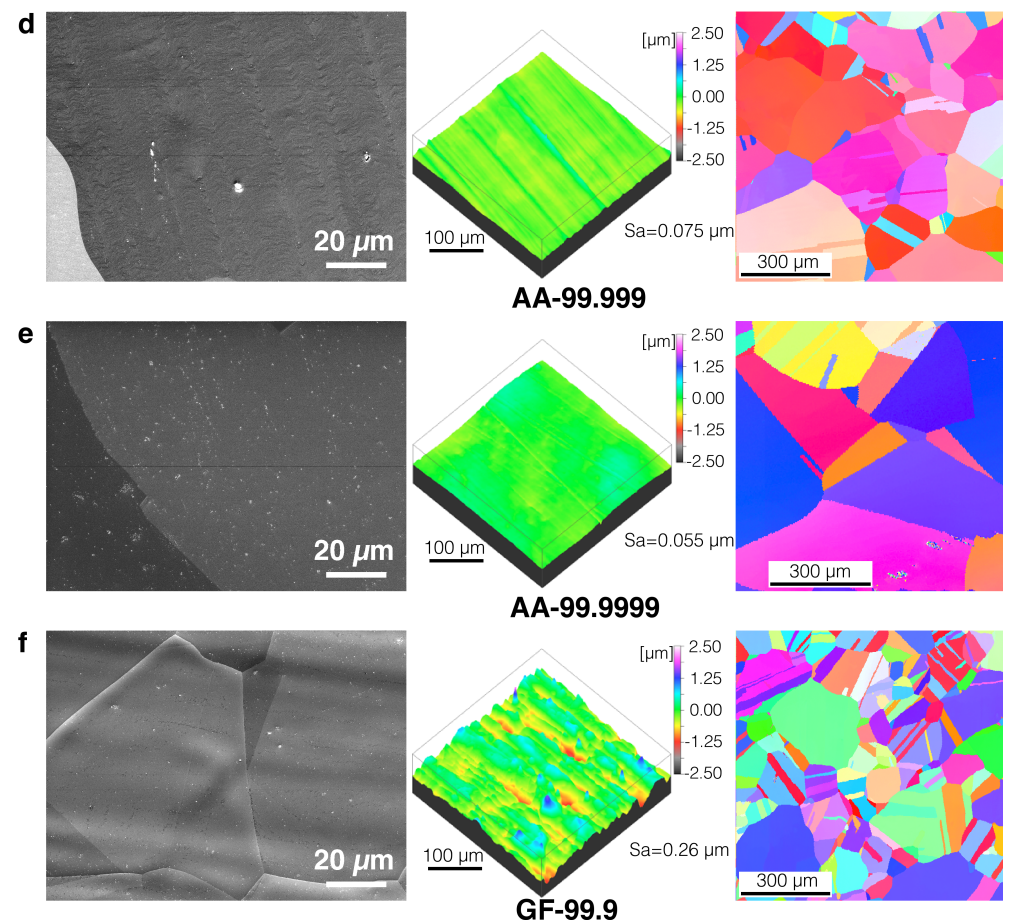

Figure 1: SEM, OP and EBSD analysis of various $\mathrm{Cu}$ foils annealed at $1035{ }^{\circ} \mathrm{C}$ for 30 minutes under $500 \mathrm{sccm}$ H2. (a) AA-99.8C, (b) AA-99.8U, (c) AA-99.99, (d) AA-99.999, (e) AA-99.9999, (f) GF-99.9. Some components of this figure are 
reprinted with permission from Murdock et al [20]. Copyright 2013 American Chemical Society. 
Surface impurities are visible on all foils, with no clear correlation between the amount observed and the supplier-stated purity, which may arise through annealing as some species segregate to the surface, while others preferentially dissolve. Processing and packaging of the foils may also be influential; indeed, the highest density of particles is visible on "coated" AA-99.8C, significantly more than its "uncoated" counterpart, AA-99.8U.

XPS unsurprisingly detected the presence of $\mathrm{Cu}, \mathrm{C}$ and $\mathrm{O}$ on all as-received foils after exposure to the atmosphere and adventitious contamination from absorbed species. $\mathrm{Ca}$, $\mathrm{P}$ and $\mathrm{Cr}$ were also detected on $\mathrm{AA}-99.8 \mathrm{C}(4.4 \%, 4.9 \%, 2.1 \%$, respectively) (Figure 2a) while Si was detected on AA-99.999 (2.7\%) and AA-99.9999 (1.6\%). Depth profiling XPS (through Ar ion bombardment) indicated that $\mathrm{Ca}, \mathrm{P}$ and $\mathrm{Cr}$ were present on AA-99.8C to a depth of ca. $5 \mathrm{~nm}$, with increased concentrations to a depth of ca. $2 \mathrm{~nm}(10.5 \%, 7.3 \%$, and $5.2 \%$, respectively). This is an indication of a surface coating on the sample of as-received AA-99.8C. After annealing treatment, $2.5 \% \mathrm{Ca}$ was still detected on AA-99.8C whilst other impurities disappeared. We confirmed that surface composition of multiple samples of the same $\mathrm{Cu}$ foil varied across the entire $30 \mathrm{~cm}$ x $30 \mathrm{~cm} \mathrm{Cu}$-foil37. As such, more thorough analysis would be required for a fully representative sampling of the entire foil. Nonetheless, XPS shows the variation in composition of the six $\mathrm{Cu}$-foil surfaces in line with observations of surface contamination particles.

SIMS confirmed the spatial distribution and elemental composition of surface impurities on the $\mathrm{Cu}$ foils. $\mathrm{C}, \mathrm{O}, \mathrm{Ca}$, and $\mathrm{Na}$ were detected on all the foils after annealing. A notably high and well-distributed Ca signal was detected on annealed AA-99.8C (Figure 2b), indicated by a high Ca:Ga ratio when compared with the other $\mathrm{Cu}$ foils (Figure 2c). Spatial SIMS imaging of the $\mathrm{Ca}$ and $\mathrm{Na}$ characteristic signals 
showed a distribution as sub-micron or micron-sized particles, corresponding with SEM observations. The spatial distribution of the Ca signal also correlated with the $\mathrm{O}$ signal (Figure $2 \mathrm{~b}$ inset), suggesting $\mathrm{Ca}$ is present as an oxide. Elemental depth profiling of annealed AA-99.8C demonstrated that Ca was present as a surface coating on the foil to a depth of $\sim 10 \mathrm{~nm}$ (Figure $2 \mathrm{~d}$ ).
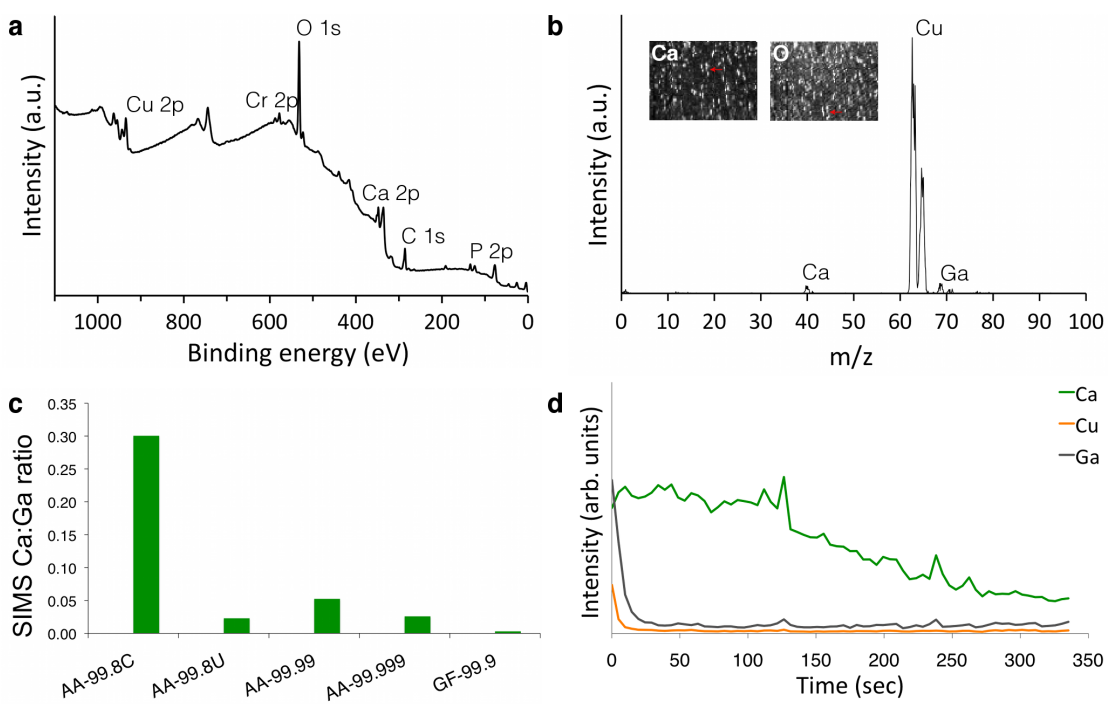

Figure 2: (a) XPS analysis of as-received AA-99.8C detecting $\mathrm{Ca}, \mathrm{Cr}$ and $\mathrm{P}$. (b) SIMS analysis (positive ions) of annealed AA-99.8 C detecting Ca. Inset (300 $\mu \mathrm{m}$ field of view) showing SIMS imaging of $\mathrm{Ca}(\mathrm{m} / \mathrm{z}=40)$ and $\mathrm{O}(\mathrm{m} / \mathrm{z}=16)$ surface contaminations (red arrows indicates corresponding particles as $\mathrm{Ca}$ and $\mathrm{O}$ scans are vertically translated). (c) SIMS analysis $\mathrm{Ca}$ : $\mathrm{Ga}$ ratio for the annealed $\mathrm{Cu}$ foils, indicating relative Ca concentration. (d) Depth profiling SIMS analysis of annealed AA-99.8C over a $75 \mu \mathrm{m}$ scan detecting $\mathrm{Ca}$ for 300 seconds, corresponding to a $\sim 10 \mathrm{~nm}$ surface coating. Note that $\mathrm{Cu}$ and $\mathrm{Ga}$ signal are enhanced in native surface oxide layer. 
EDX provided highly localised analysis of the elemental composition of contamination particles on the surface. $\mathrm{Ca}, \mathrm{Al}, \mathrm{Si}, \mathrm{O}$, and $\mathrm{C}$ were detected throughout a number of particles on the $\mathrm{Cu}$ foils, Figure 3, with the exception of AA-99.999 where only $\mathrm{Si}$ and $\mathrm{O}$ were detected. There were cases where some of these elements were absent (e.g. $\mathrm{Ca} / \mathrm{Si} / \mathrm{C} / \mathrm{O}$ particles without $\mathrm{Al}$ were detected on AA-99.8C) or other species were also detected (for instance, $\mathrm{Fe}$ or $\mathrm{Mg}$ ). However, in general we found that the particles contained $\mathrm{Ca}, \mathrm{Si}, \mathrm{Al}, \mathrm{O}$ and $\mathrm{C}$.

a
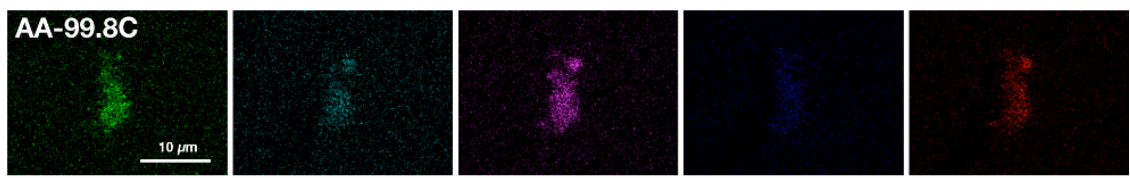

b
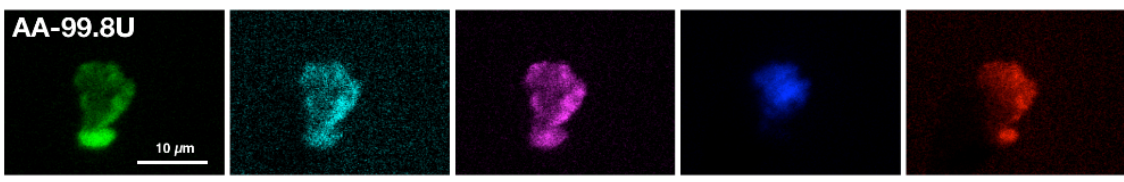

c
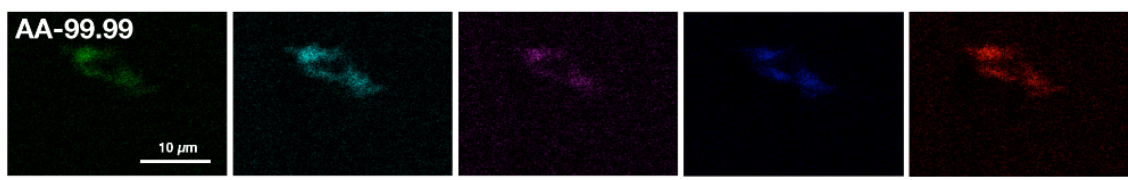

d
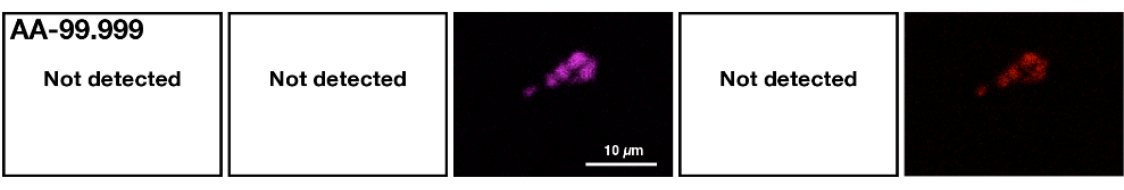

e
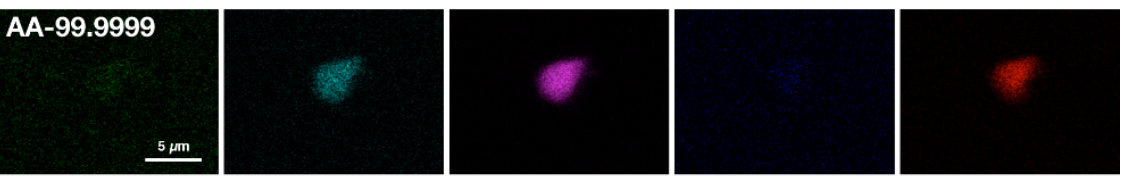

$\mathbf{f}$

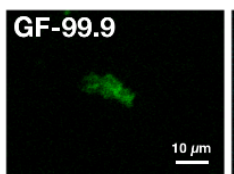

Ca

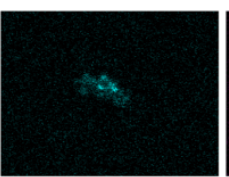

Al

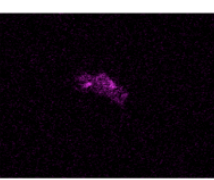

Si

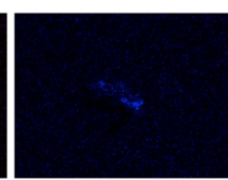

C

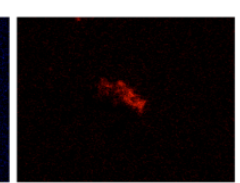

0

Figure 3: EDX mapping detecting $\mathrm{Ca}, \mathrm{Al}, \mathrm{Si}, \mathrm{C}$ and $\mathrm{O}$ on representative contamination particles on annealed $\mathrm{Cu}$ foils (a) AA-99.8C, (b) AA-99.8U, (c) AA 99.99, (d) AA-99.999, (e) AA 99.9999, (f) GF 99.9. 
Each of the six $\mathrm{Cu}$ foils from Table 1 possess a unique combination of properties, summarised in Table 2. XPS and SIMS show that the concentration of surface impurities did not decrease as the supplier's stated percentage purity improved from $99.8 \%$ up to $99.9999 \%$, as might have been expected, indicating that the surface composition differs from the bulk composition, and that any perceived benefit of using a higher purity foil to reduce the concentration of surface contaminants is erroneous. Low purity $\mathrm{Cu}$ foils sourced from different supplier showed different surface composition, which further highlights the difficult task and vigilance required if selecting $\mathrm{Cu}$ foils with specific composition requirements. The variability of properties between different $\mathrm{Cu}$ foils, and between multiple samples of one $\mathrm{Cu}$ foil, demonstrates the need for representative samples with consistent properties to overcome the risk of spurious results. The confirmation of a surface coating on AA-99.8C raises some concern about the use of this foil both with and without significant cleaning procedures [43-45].

Table 2: Combined summary of XPS, SIMS and EDX elemental surface composition of various $\mathrm{Cu}$ foils.

\begin{tabular}{|c|c|c|}
\hline Label & $\begin{array}{c}\text { As-received } \\
\text { (\%) }\end{array}$ & $\begin{array}{c}\text { Annealed } \\
\text { (\%) }\end{array}$ \\
\hline AA-99.8C & $\mathrm{C}, \mathrm{O}, \mathrm{Ca}(4.4), \mathrm{P}(4.9), \mathrm{Cr}(2.1), \mathrm{Na}, \mathrm{Cl}$ & $\mathrm{C}, \mathrm{O}, \mathrm{Ca}(2.5), \mathrm{Na}, \mathrm{Cl}, \mathrm{Si}, \mathrm{Al}$ \\
\hline $\mathrm{AA}-99.8 \mathrm{U}$ & $\mathrm{C}, \mathrm{O}, \mathrm{Ca}, \mathrm{Na}, \mathrm{K}$ & $\mathrm{C}, \mathrm{O}, \mathrm{Ca}, \mathrm{Na}, \mathrm{K}, \mathrm{Si}, \mathrm{Al}$ \\
\hline $\mathrm{AA}-99.99$ & $\mathrm{C}, \mathrm{O}, \mathrm{Ca}, \mathrm{Na}$ & $\mathrm{C}, \mathrm{O}, \mathrm{Ca}, \mathrm{Na}, \mathrm{Si}, \mathrm{Al}$ \\
\hline $\mathrm{AA}-99.999$ & $\mathrm{C}, \mathrm{O}, \mathrm{Si}(2.7), \mathrm{Ca}, \mathrm{Na}, \mathrm{Al}$ & $\mathrm{C}, \mathrm{O}, \mathrm{Ca}, \mathrm{Na}, \mathrm{Si}$ \\
\hline AA-99.9999 & $\mathrm{C}, \mathrm{O}, \mathrm{Si}(1.6), \mathrm{Ca}, \mathrm{Na}$ & $\mathrm{C}, \mathrm{O}, \mathrm{Ca}, \mathrm{Na}, \mathrm{Si}$ \\
\hline GF-99.9 & $\mathrm{C}, \mathrm{O}, \mathrm{Ca}, \mathrm{Na}$ & $\mathrm{C}, \mathrm{O}, \mathrm{Ca}, \mathrm{Na}, \mathrm{Si}, \mathrm{Al}$ \\
\hline
\end{tabular}

\subsection{Synthesis of APCVD graphene on Cu foils}

Successful synthesis of APCVD graphene on $\mathrm{Cu}$ foils was confirmed by Raman spectroscopy (Figure S1) and SEM imaging (Figure 4). The SEM images demonstrate that the graphene growth rate, nucleation density, and multilayer prevalence differs significantly between the six $\mathrm{Cu}$ foils, even though each foil was subjected to the same 
synthesis conditions. However, no clear trend is visible for the variation of graphene growth with increasing supplier-stated purity from $99.8 \%$ to $99.9999 \%$ (comparing Figure $4 \mathrm{~b}, \mathrm{~d}, \mathrm{e}, \mathrm{f})$. Furthermore, for the three foils that had equivalent purity (AA-99.8C, AA-99.8U, GF-99.9) the graphene growth was not identical, with higher nucleation density on AA-99.8C (comparing Figure 4a, b, c). Given that the surface roughness, contaminations, and texture vary between these six $\mathrm{Cu}$ foils, the influence of each factor will occur simultaneously. However, as we observe that graphene growth is relatively uniform across the entire surface of each $\mathrm{Cu}$ foil (compared to when comparing the growth on two different $\mathrm{Cu}$ foils), it is unlikely that surface topography or texture make a significant contribution (as these properties vary across the surface, for example, from grain-to-grain).
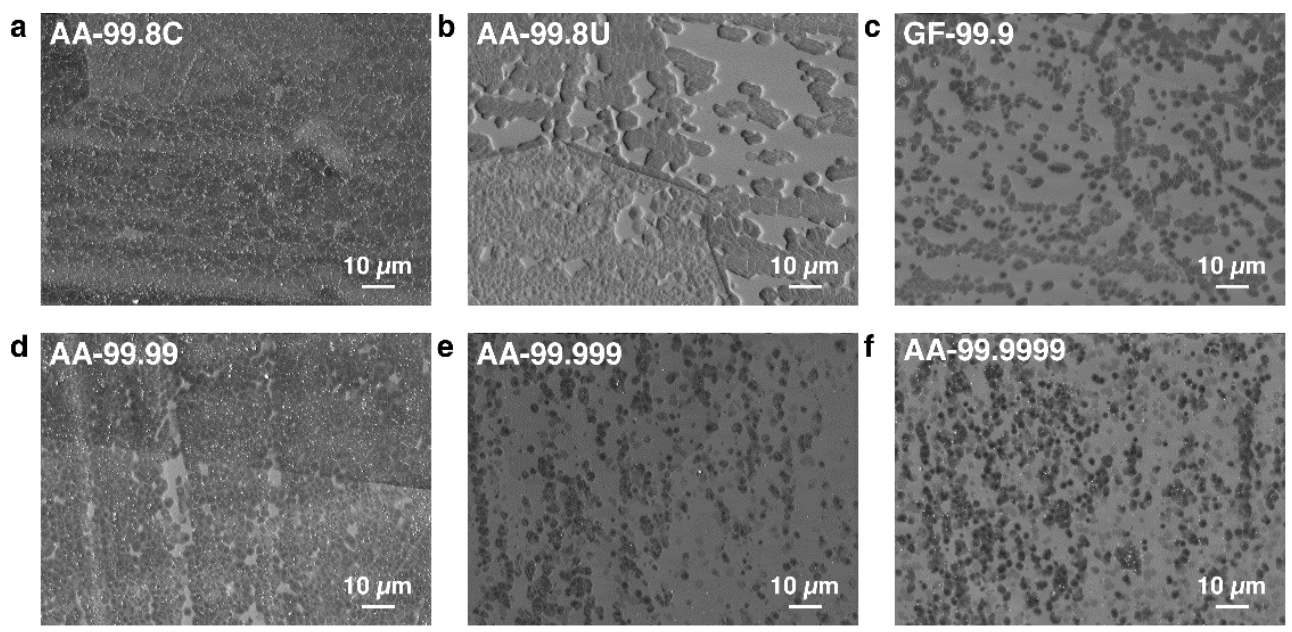

Figure 4: SEM images of APCVD graphene growth following 15 minutes synthesis at $1035{ }^{\circ} \mathrm{C}$ with $5 \mathrm{sccm} \mathrm{CH} 4$ and $300 \mathrm{sccm} \mathrm{H} 2$ simultaneously on six untreated $\mathrm{Cu}$ foil
(a) AA-99.8C,
(b) AA-99.8U,
(c) GF-99.9,
(d) AA-99.99,
(e) AA-99.999,
(f), AA-99.9999 
We therefore propose that surface composition, and particularly the presence of metallic contamination particles, significantly increase the nucleation density and growth rate of graphene on different $\mathrm{Cu}$ foils. This proposition is supported by our observations, most notably the stark variation in graphene formed on AA-99.8C and AA-99.8U (Figure $4 \mathrm{a}, \mathrm{b}$ ), which can be attributed to an influence of the $\mathrm{Ca} / \mathrm{Cr} / \mathrm{P}$ surface coating that was detected by XPS and SIMS analysis. Based on these findings we reiterate the importance of thorough characterisation of $\mathrm{Cu}$ foils prior to graphene synthesis, and recommend using identical $\mathrm{Cu}$ foils when actively following protocols from the literature.

In considering a mechanism for the cause of these observations, theoretically the contamination particles may (i) act as sites where the nucleation of graphene from (already present) surface active carbon species has a lower activation energy, or (ii) act as sites where decomposition of $\mathrm{CH}_{4}$ is increased by providing a lower decomposition activation energy, or (iii) perform both functions to varying degrees. Given that we observe nucleation without visible contamination particles, and also observe contamination particles without graphene nuclei, we suggest that most contamination particles do not primarily act as the location of nucleation sites (in agreement with previous work by Fan et al [14]).

Instead, we hypothesise that the particles increase the density of nucleation sites by increasing decomposition of $\mathrm{CH}_{4}$ and consequently increasing the concentration of active $\mathrm{C}$ species on the $\mathrm{Cu}$ surface. This hypothesis is supported by two specific scenarios we have observed: (i) increased nucleation densities surrounding large contamination particles but with nucleation sites corresponding with topographical striations, and (ii) increased growth rate of sharp lobes of graphene domains within the 
vicinity of contamination particles, which typically terminate with a particle at the apex (Figure S2).

\subsection{Treatment of $\mathrm{Cu}$ foils}

Given that the growth of graphene differed between the $\mathrm{Cu}$ foils due to their properties, in particular the concentration of surface contaminations, a natural question then arises: can chemical treatments be used to remove surface contaminations from the $\mathrm{Cu}$ foils, and so control and improve the quality of the graphene? With this in mind, we investigated the influence of different chemical treatments on the quantity and type of impurities present on the $\mathrm{Cu}$ foils and the the variation in graphene growth between as-received and chemically treated annealed substrates. Investigations were conducted using all six $\mathrm{Cu}$ foils, but owing to their popularity we primarily present data for cleaning treatments of AA-99.8C and AA-99.8U.

\subsection{1 $\quad \mathrm{CH}_{3} \mathrm{COOH}$ treatment}

$\mathrm{CH}_{3} \mathrm{COOH}$ treatment followed by annealing caused no significant difference to the surface topography for all the $\mathrm{Cu}$ foils (Figure S3). Importantly though, a reduction in surface contamination particles was observed by SEM and confirmed analytically through XPS and SIMS. After $\mathrm{CH}_{3} \mathrm{COOH}$ treatment $\mathrm{Ca}, \mathrm{P}$, and $\mathrm{Cr}$ were no longer detected by XPS on AA-99.8C, and Si was no longer detected on AA-99.999 and AA99.9999, demonstrating reduction of levels below $\sim 0.2$ atomic percent. SIMS detected reduced concentration traces of $\mathrm{Ca}$ on all six $\mathrm{CH}_{3} \mathrm{COOH}$ treated $\mathrm{Cu}$ foils (Figure $\mathrm{S} 4$ ) while Na was no longer detected on AA-99.8U and GF-99.9. We therefore conclude that $\mathrm{CH}_{3} \mathrm{COOH}$ treatment is only partially effective at reducing the concentration of nanoparticles contaminations on the surface of $\mathrm{Cu}$ foils, as some contaminations remain. Irrespective, by comparing graphene formed on $\mathrm{CH}_{3} \mathrm{COOH}$ treated (Figure S5) and untreated (Figure 4) $\mathrm{Cu}$ foils, the influence of the partial removal of 
contaminations is evident as fewer, larger domains form on the $\mathrm{CH}_{3} \mathrm{COOH}$ treated $\mathrm{Cu}$ foils for all the substrates investigated.

\subsubsection{Targeted chemical treatments $(\mathrm{HCl}, \mathrm{KOH}, \mathrm{HF}$, and combination)}

Given that XPS, SIMS and EDX analysis of the annealed $\mathrm{Cu}$ foils showed that the surface contaminations are typically composed of $\mathrm{Ca}, \mathrm{Al}, \mathrm{Si}, \mathrm{C}$ and $\mathrm{O}$, we hypothesised that purposely selected strong acids or bases may be capable of chemically dissolving these impurities. This approach is a development from previous studies that remove contaminations as a consequence of etching the surface of the $\mathrm{Cu}$ foil, rather than through targeted chemical dissolving. Due to vigorous reactions with $\mathrm{Cu}$ both concentrated nitric acid and sulphuric acid are unsuitable candidate acids. Concentrated hydrochloric acid may be suitable, as it is known to dissolve calcium and aluminium oxides:

$\mathrm{CaO}_{(\mathrm{s})}+2 \mathrm{HCl}_{(\mathrm{aq})} \rightarrow \mathrm{CaCl}_{2(\mathrm{aq})}+\mathrm{H}_{2} \mathrm{O}$

$\mathrm{Al}_{2} \mathrm{O}_{3(\mathrm{~s})}+6 \mathrm{HCl}_{(\mathrm{aq})} \rightarrow 2 \mathrm{AlCl}_{3(\mathrm{aq})}+3 \mathrm{H}_{2} \mathrm{O}$

Alternatively, concentrated $\mathrm{KOH}$ is known to dissolve aluminium oxides and silicon dioxide:

$\mathrm{Al}_{2} \mathrm{O}_{3(\mathrm{~s})}+2 \mathrm{KOH}_{(\mathrm{aq})} \rightarrow 2 \mathrm{KAl}\left(\mathrm{OH}_{4}\right)_{(\mathrm{aq})}$

$\mathrm{SiO}_{2(\mathrm{~s})}+2 \mathrm{KOH}_{(\mathrm{aq})} \rightarrow \mathrm{K}_{2} \mathrm{SiO}_{3(\mathrm{aq})}+\mathrm{H}_{2} \mathrm{O}$

Hydrofluoric acid is a final possible treatment as it is capable of dissolving calcium, silicon and aluminium oxides:

$\mathrm{CaO}_{(\mathrm{s})}+2 \mathrm{HF}_{(\mathrm{aq})} \rightarrow \mathrm{CaF}_{2(\mathrm{aq})}+\mathrm{H}_{2} \mathrm{O}$

$\mathrm{SiO}_{2(\mathrm{~s})}+4 \mathrm{HF}_{(\mathrm{aq})} \rightarrow \mathrm{SiF}_{4(\mathrm{~g})}+2 \mathrm{H}_{2} \mathrm{O}$

$\mathrm{Al}_{2} \mathrm{O}_{3(\mathrm{~s})}+6 \mathrm{HF}_{(\mathrm{aq})} \rightarrow 2 \mathrm{AlF}_{3(\mathrm{aq})}+3 \mathrm{H}_{2} \mathrm{O}$

In addition, $\mathrm{HCl}, \mathrm{KOH}$, and $\mathrm{HF}$ are not expected to react with the $\mathrm{Cu}$ foil (though $\mathrm{HCl}$ is known to dissolve copper oxides) which means the substrate can be reused. 
The effect of the targeted chemical treatments is compared with the established etching treatment of electropolishing in Figure 5. 

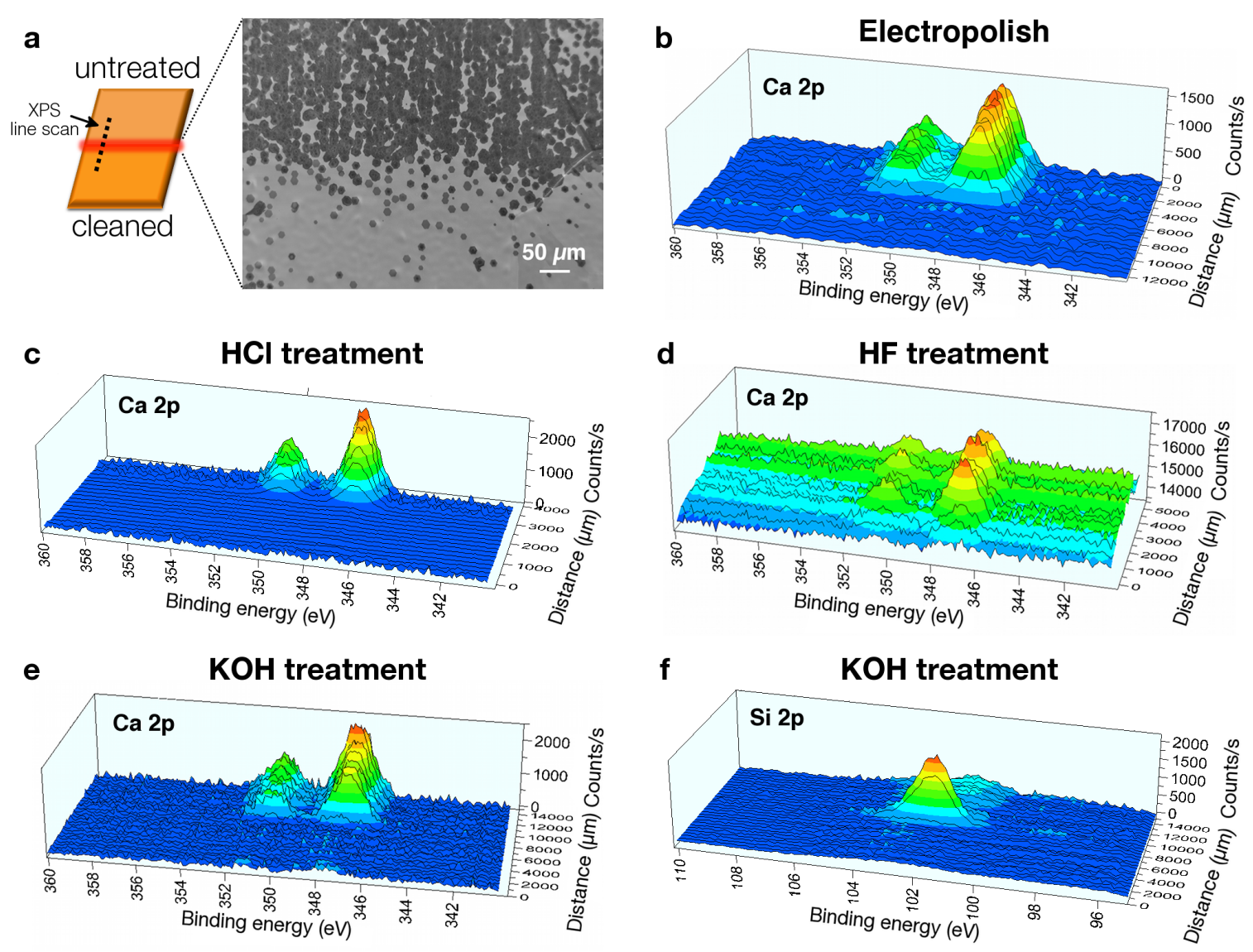

Figure 5: Effect of targeted chemical treatments on concentration of contamination particles on $\mathrm{Cu}$ foils. (a) Schematic of $\mathrm{Cu}$ foil with untreated, cleaned, and boundary region (red line) labelled, alongside SEM demonstrating variation of APCVD growth of graphene over such an electropolished boundary on AA-99.8U. (b) Detailed XPS Ca $2 p$ line scan across electropolished boundary on AA-99.8U. (c) Detailed XPS Ca $2 \mathrm{p}$ line scan across $\mathrm{HCl}$ treatment boundary on AA-99.8U. (d) Detailed XPS Ca 2p line scan across HF treatment boundary on AA-99.8C. (e) Detailed XPS Ca $2 p$ line scan over $\mathrm{KOH}$ treatment boundary on AA-99.8C. (f) Detailed XPS Si 2p line scan over $\mathrm{KOH}$ treatment boundary on AA-99.8C. 
A clear reduction in XPS Ca $2 p$ signal intensity across the electropolishing boundary correlates with a reduction in the nucleation of graphene domains (Figure $5 \mathrm{a}$ and $5 \mathrm{~b}$ ). Similarly, a reduction of the $\mathrm{Ca} 2 \mathrm{p}$ signal intensity across the $\mathrm{HCl}$ and $\mathrm{HF}$ treatment boundaries (Figure 5c and 5d) and a reduction of the Si2p signal intensity (Figure 5f) and the $\mathrm{Ca} 2 \mathrm{p}$ signal intensity across the $\mathrm{KOH}$ treatment boundary (Figure 5e) demonstrates the effectiveness of the targeted cleaning treatments. We rationalize this latter observation by recognising that chemically targeting one species (e.g. Si) within a $\mathrm{Ca} / \mathrm{Si} / \mathrm{Al} / \mathrm{C} / \mathrm{O}$ compound may in fact remove the entire particle, including untargeted species (e.g. Ca). The XPS line scan analysis is supported by a reduction in the amount of contamination particles visible by $\mathrm{SEM}$ after $\mathrm{HCl}, \mathrm{KOH}$ and $\mathrm{HF}$ treatment (Figure S6), while EDX mapping of particles still present on $\mathrm{HCl}$ treated $\mathrm{Cu}$ foils showed they were composed of $\mathrm{Si}, \mathrm{Al}$ and $\mathrm{O}$, but significantly, no Ca was detected (Figure S7). These results demonstrate that $\mathrm{HCl}$ treatment is an effective method of dissolving $\mathrm{Ca}$ contaminations from the surface of $\mathrm{Cu}$ foils (although $\mathrm{Al}$ and $\mathrm{Si}$ contaminations persist), while $\mathrm{KOH}$ treatment is an effective method of dissolving $\mathrm{Ca}, \mathrm{Al}$ and $\mathrm{Si}$ contaminations. We therefore propose a combination cleaning using $\mathrm{HCl}$ and $\mathrm{KOH}$, aiming to remove $\mathrm{Ca}, \mathrm{Al}$ and $\mathrm{Si}$ through the complementary reactivity of the chemicals.

Simultaneous APCVD growth of graphene on $\mathrm{HCl}, \mathrm{HF}, \mathrm{KOH}$, and combined $\mathrm{HCl} / \mathrm{KOH}$ treated $\mathrm{Cu}$ foils demonstrates that the targeted treatments are effective at reducing graphene nucleation density when compared to untreated $\mathrm{Cu}$ foils (Figure 6). SEM imaging demonstrates the greatest improvements using the combination $\mathrm{HCl} / \mathrm{KOH}$ treatment, with a lower nucleation density than on $\mathrm{HNO}_{3}$ treated $\mathrm{Cu}$ foils, and only a slightly higher nucleation density than electropolished $\mathrm{Cu}$ foils. 


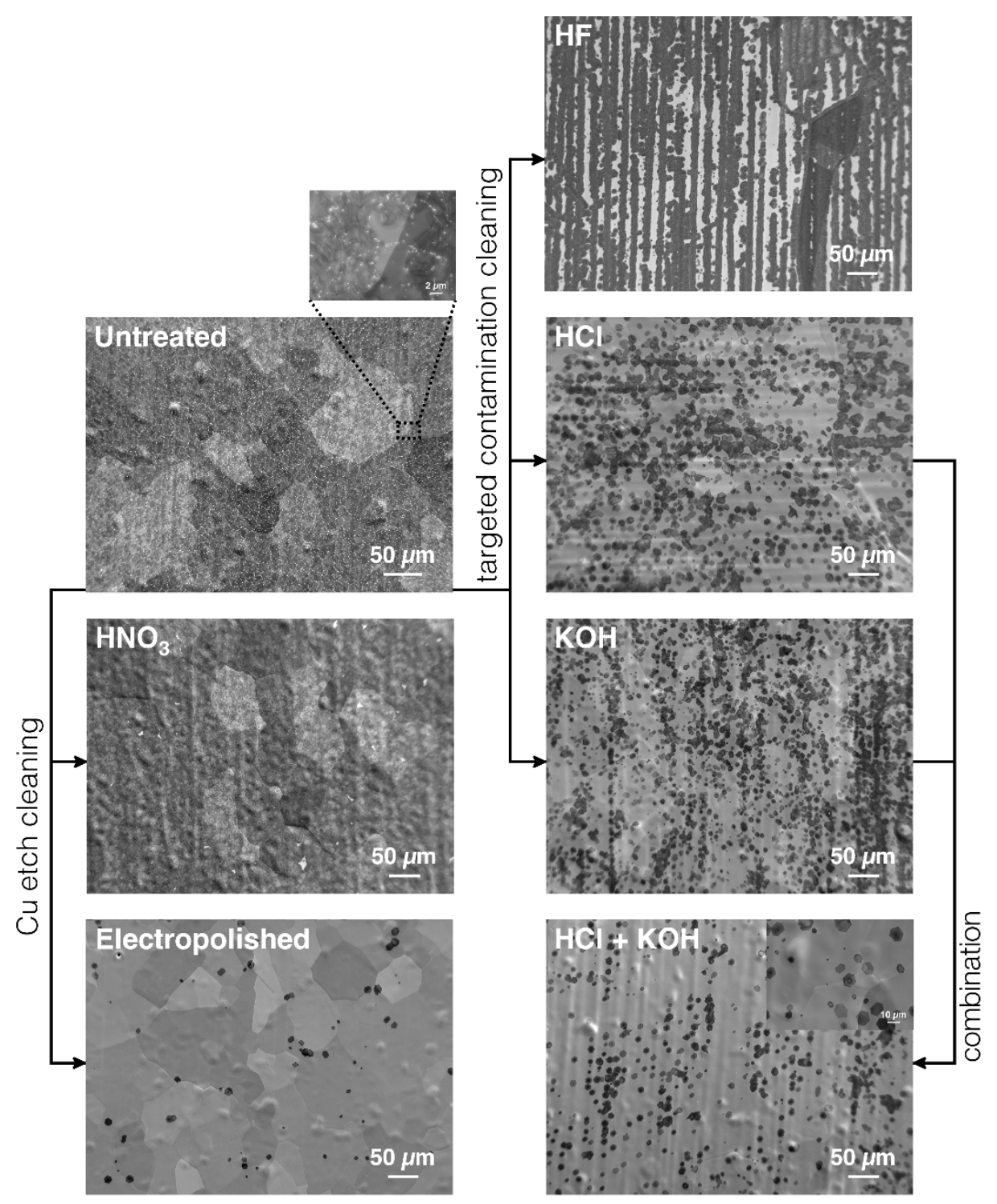

Figure 6: Effect of targeted chemical treatments of $\mathrm{Cu}$ foils on APCVD graphene growth. Graphene domain nucleation density is reduced through targeted contamination cleaning ( $\mathrm{HF}, \mathrm{HCl}, \mathrm{KOH}$, and combined $\mathrm{HCl} / \mathrm{KOH}$ treatment) when compared to untreated and $\mathrm{HNO}_{3}$ treated $\mathrm{Cu}$ foils. Combination $\mathrm{HCl} / \mathrm{KOH}$ treatment is most effective, providing similar improvements to that of the harsher and more complicated electropolished treatment. 


\section{Conclusions}

In this article we presented a detailed analysis of the surface impurities of $\mathrm{Cu}$ foils sourced from different suppliers and with varying purities, with the goal of improving the growth of APCVD graphene. We show that surface contamination particles composed of calcium, aluminium and silicon oxides were present on $\mathrm{Cu}$ foils with purities ranging between $99.8 \%$ and $99.9999 \%$ and that these particles influence the nucleation density, growth rate, and growth features of graphene domains. The concentration of surface impurities did not decrease as the supplier's stated percentage purity improved, as might have been expected, and with regards to the prototypical $\mathrm{Cu}$ foil, $99.8 \%$ Alfa Aesar item 13382, we demonstrated that this is by no means an ideal substrate as it possesses a $\sim 5-10 \mathrm{~nm}$ surface coating of $\mathrm{Ca}$ and $\mathrm{Cr}$ as-received from the supplier. Based on the analytical characterisation of the $\mathrm{Cu}$ foils we proposed chemical treatments to dissolve the surface contamination particles using $\mathrm{HCl}, \mathrm{KOH}, \mathrm{HF}$, and a combination of $\mathrm{HCl} / \mathrm{KOH}$ treatments. By targeted removal of the copper foil surface impurities the growth of APCVD graphene was improved with reduced nucleation density and less nanoparticle contamination. These new solution-based cleaning treatments are therefore fast and effortless, can easily be scaled and further improved by repeated cycling of treatments and annealing, are compatible with roll-to-roll processing, and leave the $\mathrm{Cu}$ foil undamaged for reuse, making them attractive procedures for improving the synthesis of large-area CVD graphene.

\section{Acknowledgements}

We are grateful for support from the Commonwealth Scholarship Commission (A.T.M.), University of Oxford Clarendon Fund (A.T.M.), the Royal Society (N.G.), the European Research Council (ERC-2009-StG-240500) (N.G.), the Engineering and 
Physical Sciences Research Council Pathways to Impact Award (N.G.) and The Korea Institute of Energy Research, KIER, (N.G., A.T.M.)

\section{References}

1. K. S. Novoselov, V. I. Fal'ko, L. Colombo, P. R. Gellert, M. G. Schwab and K. Kim, A Roadmap for Graphene. Nature, 2012, 490, 192-200.

2. A. C. Ferrari, F. Bonaccorso, V. Fal'ko, K. S. Novoselov, S. Roche, P. Boggild, et al. Science and Technology Roadmap for Graphene, Related Two-Dimensional Crystals, and Hybride Systems. Nanoscale, 2015, 7, 4598-4810.

3. J. Wintterlin and M.-L. Bocquet, Graphene on Metal Surfaces. Surf. Sci., 2009, $603,1841-1852$.

4. X. Li, W. Cai, J. An, S. Kim, J. Nah, D. Yang, et al. Large-Area Synthesis of High-Quality and Uniform Graphene Films on Copper Foils. Science, 2009, 324, 1312-1314.

5. S. Bae, H. Kim, Y. Lee, X. Xu, J.-S. Park, Y. Zheng, et al. Roll-to-Roll Production of 30-Inch Graphene Films for Transparent Electrodes. Nat. Nanotechnol., $2010,5,574-578$.

6. E. S. Polsen, D. Q. McNerny, B. Viswanath, S. W. Pattinson and A. John Hart, High Speed Roll-to-Roll Manufacturing of Graphene Using a Concentric Tube CVD Reactor. Scientific Reports, 2015, 5, 10257.

7. T. Kobayashi, M. Bando, N. Kimura, K. Shimizu, K. Kadono, N. Umezu, et al. Production of a 100-m-Long High-Quality Graphene Transparent Conductive Film by 
Roll-to-Roll Chemical Vapor Deposition and Transfer Process. Applied Physics Letters, 2013, 102, 023112.

8. Z. Luo, Y. Lu, D. W. Singer, M. E. Berck, L. A. Somers, B. R. Goldsmith and A. T. C. Johnson, Effect of Substrate Roughness and Feedstock Concentration on Growth of Wafer-Scale Graphene at Atmospheric Pressure. Chem. Mater., 2011, 23, 1441-1447.

9. G. H. Han, F. Güneş, J. J. Bae, E. S. Kim, S. J. Chae, H.-J. Shin, et al. Influence of Copper Morphology in Forming Nucleation Seeds for Graphene Growth. Nano Lett., 2011, 11, 4144-4148.

10. A. Mohsin, L. Liu, P. Liu, W. Deng, I. N. Ivanov, G. Li, et al. Synthesis of Millimeter-Size Hexagon-Shaped Graphene Single Crystals on Resolidified Copper. ACS Nano, 2013, 7, 8924-8931.

11. Y. Hao, M. S. Bharathi, L. Wang, Y. Liu, H. Chen, S. Nie, et al. The Role of Surface Oxygen in the Growth of Large Single-Crystal Graphene on Copper. Science, $2013,342,720-723$.

12. P. Braeuninger-Weimer, B. Brennan, A. J. Pollard and S. Hofmann, Understanding and Controlling Cu-Catalyzed Graphene Nucleation: The Role of Impurities, Roughness, and Oxygen Scavenging. Chemistry of Materials, 2016, 28, $8905-8915$.

13. W. Liu, H. Li, C. Xu, Y. Khatami and K. Banerjee, Synthesis of High-Quality Monolayer and Bilayer Graphene on Copper Using Chemical Vapour Deposition. Carbon, 2011, 49, 4122-4130. 
14. L. Fan, K. Wang, J. Wei, M. Zhong, D. Wu and H. Zhu, Correlation Between Nanoparticle Location and Graphene Nucleation in Chemical Vapour Deposition of Graphene, J. Mater. Chem. A, 2014, 2, 13123-13128.

15. Q. Yu, L. A. Jauregui, W. Wu, R. Colby, J. Tian, Z. Su, et al. Control and Characterization of Individual Grains and Grain Boundaries in Graphene Grown by Chemical Vapour Deposition. Nat. Mater., 2011, 10, 443-449.

16. H. Wang, G. Wang, P. Bao, S. Yang, W. Zhu, X. Xie and W.-J. Zhang, Controllable Synthesis of Submillimeter Single-Crystal Monolayer Graphene Domains on Copper Foils by Suppressing Nucleation. Journal of the American Chemical Society, 2012.

17. Y. Xiao, H. Kim, C. Mattevi, M. Chhowalla, R. C. Maher and L. F. Cohen, Influence of $\mathrm{Cu}$ Substrate Topography on the Growth Morphology of Chemical Vapour Deposited Graphene. Carbon, 2013, 65, 7-12.

18. Z. Yan, J. Lin, Z. Peng, Z. Sun, Y. Zhu, L. Li, et al. Towards the Synthesis of Wafer-Scale Single-Crystal Graphene on Copper. ACS Nano, 2012, 6, 9110-9117.

19. J. D. Wood, S. W. Schmucker, A. S. Lyons, E. Pop and J. W. Lyding, Effects of Polycrystalline $\mathrm{Cu}$ Substrate on Graphene Growth by Chemical Vapor Deposition. Nano Lett., 2011, 11, 4547-4554.

20. A. T. Murdock, A. Koos, T. B. Britton, L. Houben, T. Batten, T. Zhang, et al. Controlling the Orientation, Edge Geometry and Thickness of Chemical Vapor Deposition Graphene. ACS Nano, 2013, 7, 1351-1359. 
21. X. Li, Y. Zhu, W. Cai, M. Borysiak, B. Han, D. Chen, et al. Transfer of LargeArea Graphene Films for High-Performance Transparent Conductive Electrodes. Nano Lett., 2009, 9, 4359-4363.

22. H. Cao, Q. Yu, L. A. Jauregui, J. Tian, W. Wu, Z. Liu, et al. Electronic Transport in Chemical Vapor Deposited Graphene Synthesized on Cu: Quantum Hall Effect and Weak Localization. Appl. Phys. Lett., 2010, 96, 259901.

23. W. Regan, N. Alem, B. N. Alemán, B. Geng, C. L. Girit, L. Maserati, et al. A Direct Transfer of Layer-Area Graphene. Appl. Phys. Lett., 2010, 96, 113102.

24. W. Cai, A. L. Moore, Y. Zhu, X. Li, S. Chen, L. Shi and R. S. Ruoff, Thermal Transport in Suspended and Supported Monolayer Graphene Grown by Chemical Vapor Deposition. Nano Lett., 2010, 10, 1645-1651.

25. B. N. Alemán, W. Regan, S. Aloni, V. Altoe, N. Alem, C. L. Girit, et al. Transfer-Free Batch Fabrication of Large-Area Suspended Graphene Membranes. ACS Nano, 2010.

26. J. Cho, L. Gao, J. Tian, H. Cao, W. Wu, Q. Yu, et al. Atomic-Scale Investigation of Graphene Grown on $\mathrm{Cu}$ Foil and the Effects of Thermal Annealing. ACS Nano, 2011, 5, 3607-3613.

27. P. Y. Huang, C. S. Ruiz-Vargas, A. M. van der Zande, W. S. Whitney, M. P. Levendorf, J. W. Kevek, et al. Grains and Grain Boundaries in Single-Layer Graphene Atomic Patchwork Quilts. Nature, 2011, 469, 389-392.

28. K. Kim, Z. Lee, W. Regan, C. Kisielowski, M. F. Crommie and A. Zettl, Grain Boundary Mapping in Polycrystalline Graphene. ACS Nano, 2011, 5, 2142-2146. 
29. T. M. Paronyan, E. M. Pigos, G. Chen and A. R. Harutyunyan, Formation of Ripples in Graphene as a Result of Interfacial Instabilities. ACS Nano, 2011, 5, 96199627.

30. A.-Y. Lu, S.-Y. Wei, C.-Y. Wu, Y. Hernandez, T.-Y. Chen, T.-H. Liu, et al. Decoupling of CVD Graphene by Controlled Oxidation of Recyrstallized Cu. RSC Adv., 2012, 2, 3008.

31. L. Gan and Z. Luo, Turning of Hydrogen to Realize Seeded Growth of Subcentimeter Single-Crystal Graphene Grains on Copper. ACS Nano, 2013, 7, 94809488.

32. P. R. Kidambi, C. Ducati, B. Dlubak, D. Gardiner, R. S. Weatherup, M.-B. Martin, et al. The Parameter Space fo Graphene Chemical Vapor Deposition on Polycrystalline Cu. J. Phys. Chem. C, 2012, 116, 22492-22501.

33. J. M. Wofford, S. Nie, K. F. McCarty, N. C. Bartelt and O. D. Dubon, Graphene Islands on Cu Foils: the Interplay Between Shape, Orientation, and Defects. Nano Lett., 2010, 10, 4890-4896.

34. I. Vlassiouk, P. Fulvio, H. Meyer, N. Lavrik, S. Dai, P. Datskos and S. Smirnov, Large Scale Atmospheric Pressure Chemical Vapor Deposition of Graphene. Carbon, 2013, 54, 58-67.

35. L. Fan, J. Zou, Z. Li, X. Li, K. Wang, J. Wei, et al. Topology Evolution of Graphene in Chemical Vapor Deposition, a Combined Theoretical/Experimental Approach Toward Shape Control of Graphene Domains. Nanotechnology, 2012, 23, 115605. 
36. B. Zhang, W. H. Lee, R. Piner, I. Kholmanov, Y. Wu, H. Li et al. LowTemperature Chemical Vapor Deposition Growth of Graphene From Toluene on Electropolished Copper. ACS Nano, 2012, 6, 2471-2476.

37. S. M. Kim, A. Hsu, Y.-H. Lee, M. Dresselhaus, T. Palacios, K. K. Kim and J. Kong, The Effect of Copper Pre-Cleaning on Graphene Synthesis. Nanotechnology, $2013,24,365602$.

38. Z.-J. Wang, G. Weinberg, Q. Zhang, T. Lunkenbein, A. Klein-Hoffmann, M. Kurnatowska, et al. Durect Observation of Graphene Growth and Associated Copper Substrate Dynamics by in Situ Scanning Electron Microscopy. ACS Nano, 2015, 9, 1506-1519.

39. M. P. Levendorf, C. S. Ruiz-Vargas, S. Garg and J. Park, Transfer-Free Batch Fabrication of Single Layer Graphene Transistors. Nano Lett., 2009, 9, 4479-4483.

40. C. A. Howsare, X. Weng, V. Bojan, D. Snyder and J. A. Robinson, Substrate Considerations for Graphene Synthesis on Thin Copper Films. Nanotechnology, 2012, $23,135601$.

41. Z. Luo, S. Kim, N. Kawamoto, A. M. Rappe and A. T. C. Johnson, Growth Mechanism of Hexagonal-Shape Graphene Flakes with Zigzag Edges. ACS Nano, $2011,5,9154-9160$.

42. N. Reckinger, A. Felten, C. N. Santos, B. Hackens and J. F. Colomer, The Influence of Residual Oxidizing Impurities on the Synthesis of Graphene by Atmospheric Pressure Chemical Vapor Deposition. Carbon, 2013, 63, 84-91. 
43. Y. Wu, Y. Hao, H. Y. Jeong, Z. Lee, S. Chen, W. Jiang, et al. Crystal Structure Evolution of Individual Graphene Islands During CVD Growth on Copper Foil. Adv. Mater., 2013, 25, 6744-6751.

44. J.-Y. Lim, J.-H. Lee, H.-S. Jang, W.-J. Joo, S. Hwang and D. Whang, Selective Exfoliation of Single-Layer Graphene From Non-Uniform Graphene Grown on Cu. Nanotechnology, 2015, 26, 455304.

45. S. Zhu, Q. Li, Q. Chen, W. Liu, X. Li, J. Zhang, et al. Cu Hill and Graphene Grain Evolution in the Synthesis of Millimeter-Sized Single Crystal Graphene During Low Pressure Chemical Vapor Deposition. RSC Advances, 2014, 4, 32941-32945. 